\title{
Optimizing safety surveillance for COVID-19 vaccines
}

Rebecca E. Chandler(i)

Evolution of the current infrastructure for surveillance of vaccine safety will be essential to meet our commitments to the public in the deployment of a vaccine (or vaccines) to COVID-19. The incorporation of concepts and tools within the fields of data science and systems immunology can be used to propel vaccine safety monitoring into the twenty-first century.

Vaccines have had a profound impact on public health, with their benefits enjoyed by populations around the globe. However, even after widespread vaccine acceptance and decades of use, including in countries with high vaccination rates, public concerns over the safety of vaccines have been documented ${ }^{1}$. Citing measles outbreaks, the World Health Organization (WHO) last year declared 'vaccine hesitancy' one of ten threats to global health ${ }^{2}$.

The deployment of a vaccine (or vaccines) to COVID-19 will be ground-breaking, given the immediacy of its need in relation to the current development phase of the candidate vaccines. Some initial work on coronavirus vaccines had been completed before the COVID-19 pandemic, and challenges to the development of effective and safe vaccines against coronaviruses have been detailed in the literature ${ }^{3}$. Multiple vaccines to COVID-19 are currently being investigated, some in traditional systems using known adjuvants and others using currently unlicensed technologies ${ }^{4}$, with regulatory bodies promising to fast-track approval procedures ${ }^{5}$. The imbalance between our knowledge of the safety of a vaccine candidate and the extent of potential postapproval use of that vaccine at the time of licensure will be very large indeed.

Public confidence in vaccination programmes is therefore at high risk if the systems for monitoring vaccine safety do not perform optimally. Improved coordination and the incorporation of new methodologies and technologies into current vaccine safety systems will be crucial if we are to meet our obligations to safeguard from potential harms caused by vaccination. The COVID-19 pandemic could be the catalyst that propels vaccine safety surveillance into the twenty-first century. of pivotal trials. Assessment of safety is typically a secondary objective, and pivotal trials are not powered to support statistical analyses of end points of specific adverse events following immunization (AEFIs). The safety data collected within these trials are sufficient to characterize the more common adverse events - the local and systemic reactions related to the immunogenicity of the vaccine - that occur within a short time after vaccination. Only after the vaccine is administered within large populations after licensure is it possible to detect any rare adverse events that were not observed in clinical trials.

Monitoring of vaccine safety after licensure relies upon a combination of passive and active surveillance. Passive surveillance systems, which are the foundation of pharmacovigilance, are databases into which spontaneous reports of AEFIs are collected, such as the Vaccine Adverse Event Reporting System (VAERS) in the United States and EudraVigilance in the European Union (EU). Routine surveillance for safety signals is based upon a statistical pair-wise analysis that detects disproportionality between the number of observed reports and the number of expected reports of a single adverse event for a single vaccine (such as febrile seizure for pneumococcal vaccine), followed by clinical validation and assessment of the case series for that vaccine and that AEFI. By contrast, active surveillance systems seek to ascertain all reports of pre-specified AEFIs from a representative sample, such as sentinel sites. An advantage of such systems is that the 'denominator', or size of the population from which the AEFI arose, is known. This is followed by comparative incidence analyses of AEFIs in subpopulations who have not received the vaccine (or a pre-vaccination time period for subjects experiencing an AEFI after vaccination), using standardized case definitions and large, linked networks of health insurance claims or electronic health record data, such as Vaccine Safety Datalink (VSD) in the United States and ADVANCE (Accelerated Development of Vaccine Benefit-Risk Collaboration in Europe) in the EU. 


\section{Global variation in safety surveillance}

However, there is large variation in the capacity to carry out post-licensure vaccine safety surveillance between countries ${ }^{7}$. The system described above is largely applicable only to high-income countries. Within these countries, AEFI reports are collected in the databases of national regulatory authorities (NRAs). These reports are mainly received from physicians and patients themselves, either directly or through vaccine manufacturers. Within such databases, the detection of safety signals and causality assessment are approached in a similar manner for all medicinal products. Furthermore, advanced diagnostics and electronic health-care data sources are available to carry out epidemiological studies for signal evaluation and/or active surveillance studies using standardized case definitions.

By contrast, within many low-income and middleincome countries, vaccines are largely administered by national immunization centres, which are also responsible for collecting data on AEFIs. Support to the national immunization programmes for safety surveillance has been provided by the Global Vaccine Safety Blueprint (GVSB) of the $\mathrm{WHO}^{8}$. The tools and guidance provided through the GVSB prioritize the detection of AEFIs that are suggestive of programmatic errors, such as immunization errors or quality-related problems, and of well-characterized AEFIs that are known to occur rarely after certain vaccines, such as thrombocytopenia or intussusception. In these countries, NRAs capture a smaller number of AEFI reports, such as those reported directly by health-care providers and/or reports transferred to them by vaccine manufacturers. Only reports handled by NRAs are shared with VigiBase, the global database of the WHO Programme for International Drug Monitoring. Furthermore, limited resources within the health-care systems of these countries inhibit the ability to routinely carry out surveillance beyond passive reporting into spontaneous databases, and the separation of AEFI reports between national immunization centres and NRAs is suboptimal.

\section{Global coordination and innovation needed}

To ensure the safety of COVID-19 vaccines, global cooperation in a robust system for the timely detection and elucidation of any safety signals will be crucial. Real-time global data exchange is essential as the pooling of reports of AEFIs into larger databases will allow for the earlier detection of safety signals. Any gaps in communication between local, regional and international public health authorities handling AEFI data must be filled, and any weaknesses in existing networks of health data must be strengthened. A roadmap for international collaborative epidemiological monitoring of vaccine safety in low-income and middle-income countries has been described and should be put into practice as a matter of urgency ${ }^{9}$.

Preparation for 'AEFI X', the unexpected adverse event, requires the consideration of different approaches to signal detection. New vaccine technologies, such as those being explored for COVID-19 vaccines, could cause AEFIs that are more complex and difficult to recognize. Approaches such as syndromic surveillance could be considered; this was originally developed for the early detection of release of a biological weapon with an objective to identify illness clusters early, before diagnoses are confirmed, and to mobilize a rapid response. Elucidation of the mechanisms by which vaccines might cause harm requires collaboration with immunologists. With the growing evidence of inter-individual variation in vaccine responses based on differences in innate immunity, microbiomes and immunogenetics, there is an emerging field within vaccinology, known as adversomics, that acknowledges that AEFIs might be individually determined ${ }^{10}$. If and when a vaccine safety concern is identified, a commitment by regulatory authorities to determine how and why an AEFI, however rare, occurred is essential both to further our knowledge of the immune system and to ensure public trust in immunization programmes.

\footnotetext{
1. Vaccine Confidence Project. State of vaccine confidence in the EU: 2018. Vaccine Confidence https://www.vaccineconfidence.org/ research-feed/the-state-of-vaccine-confidence-in-the-eu-2018 (2018).

2. World Health Organization. Ten threats to global health in 2019. WHO https://www.who.int/news-room/feature-stories/ten-threatsto-global-health-in-2019 (2019).

3. Hotez, P. J., Corry, D. B. \& Bottazzi, M. E. COVID-19 vaccine design: the Janus face of immune enhancement. Nat. Rev. Immunol. 20 , 347-348 (2020)

4. Thanh, Le,T. et al. The COVID-19 vaccine development landscape. Nat. Rev. Drug Discov. 19, 305-306 (2020).

5. European Medicines Agency, COVID-19: how EMA fast-tracks development support and approval of medicines and vaccines. EMA https://www.ema.europa.eu/en/news/covid-19-how-ema-fasttracks-development-support-approval-medicines-vaccines (2020).

6. World Health Organization. Annex 9: guidelines on clinical evaluation of vaccines: regulatory expectations. WHO https:// www.who.int/biologicals/expert_committee/WHO_TRS_1004_ web_Annex_9.pdf?ua=1 (2017).

7. World Health Organization. Global vaccine safety blueprint 2.0 background research. WHO https://www.who.int/vaccine_safety/ publications/2019_Landscape_Analysis.pdf?ua=1 (2019).

8. World Health Organization. Global vaccine safety blueprint. WHO https://apps.who.int/iris/handle/10665/70919 (2012).

9. Izurieta, H. S. et al. Roadmap for the international collaborative epidemiologic monitoring of safety and effectiveness of new high priority vaccines. Vaccine 31, 3623-3627 (2013).

10. Poland, G. A., Ovsyannikova, I. G. \& Jacobson, R. M. Adversomics: the emerging field of vaccine adverse event immunogenetics. Pediatr. Infect. Dis. J. 358, 431-432 (2009).
}

Competing interests

The author declares no competing interests. 